\title{
On post quantum estimates of upper bounds involving twice $(p, q)$-differentiable preinvex function
}

\author{
Muhammad Uzair Awan', Sadia Talib', Muhammad Aslam Noor², Yu-Ming Chu* (D) and \\ Khalida Inayat Noor ${ }^{2}$
}

"Correspondence:

chuyuming2005@126.com

${ }^{3}$ Department of Mathematics,

Huzhou University, Huzhou, China

Full list of author information is

available at the end of the article

\section{Springer}

\begin{abstract}
The main objective of this paper is to derive a new post quantum integral identity using twice $(p, q)$-differentiable functions. Using this identity as an auxiliary result, we obtain some new post quantum estimates of upper bounds involving twice $(p, q)$-differentiable preinvex functions.
\end{abstract}

MSC: 26A51;26D15;05A30

Keywords: Convex; Preinvex; $(p, q)$-calculus; Bounds; Inequalities

\section{Introduction and preliminaries}

The quantum calculus is often regarded as calculus without limits, we obtain $q$-analogues of mathematical objects which can be recaptured by taking $q \rightarrow 1^{-}$. Historically the subject of quantum calculus can be traced back to Euler and Jacobi, but in recent decades it has experienced a rapid development. This can be attributed to the fact that it serves as a bridge between mathematics and physics. It is also pertinent to mention here that quantum calculus is a subfield of time scale calculus. In quantum calculus, we are concerned with a specific time scale, called the $q$-time scale. In the twentieth century Jackson [8] introduced the notion of $q$-definite integrals in quantum calculus. This motivated many quantum calculus analysts, and consequently a number of articles have been written in this area. It is worth to mention here for interested readers that it is possible that sometimes more than one $q$-analogue exists. In [9] interested readers may find some basic and interesting details on some recent developments of basic theory of quantum calculus. While studying quantum calculus, Tariboon et al. [23] introduced the notions of $q$-derivatives and $q$-integrals on finite intervals and developed several new $q$-analogues of classical inequalities. This particular article inspired many researchers working in the field of inequalities, particulary inequalities involving convexity and its generalizations. Resultantly, several new quantum analogues of classical results have been obtained. For example, Noor et al. [21] obtained the quantum analogues of Hermite-Hadamard's inequality using the class of preinvex functions. Sudsutad et al. [22] and Noor et al. [20]

C) The Author(s) 2020. This article is licensed under a Creative Commons Attribution 4.0 International License, which permits use, sharing, adaptation, distribution and reproduction in any medium or format, as long as you give appropriate credit to the original author(s) and the source, provide a link to the Creative Commons licence, and indicate if changes were made. The images or other third party material in this article are included in the article's Creative Commons licence, unless indicated otherwise in a credit line to the material. If material is not included in the article's Creative Commons licence and your intended use is not permitted by statutory regulation or exceeds the permitted use, you will need to obtain permission directly from the copyright holder. To view a copy of this licence, visit http://creativecommons.org/licenses/by/4.0/. 
obtained new quantum analogues of trapezium like inequalities involving $q$-differentiable convex functions. Noor et al. [19] obtained quantum analogues of Ostrowski's inequality. Zhang et al. [26] obtained a new generalized $q$-integral identity, and utilizing this as an auxiliary result, they have obtained several new $q$-analogues of classical inequalities. Liu and Zhuang [15] obtained certain new $q$-analogues of Hermite-Hadamard's inequality using two times $q$-differentiable convex functions. Alp et al. [3] obtained some new refined $q$-analogues of Hermite-Hadamard's inequality. For more details, see $[4,10,11,13]$

A recent development in the study of quantum calculus is the introduction of post quantum calculus. In quantum calculus we deal with $q$-number with one base $q$; however, post quantum calculus includes $p$ and $q$-numbers with two independent variables $p$ and $q$. This was first considered by Chakarabarti and Jagannathan [7]. For some interesting applications, see $[1,2,6,12,17,18]$. Motivated by the research work going on, Tunc and Gov [24] introduced the concepts of $(p, q)$-derivatives and $(p, q)$-integrals on finite intervals.

Since the appearance of this article, a number of new post quantum analogues of classical inequalities have been obtained. For example, Kunt et al. [14] obtained new post quantum analogues of Hermite-Hadamard's inequality. Luo et al. [16] obtained some new variants of parameterized $(p, q)$-integral inequalities using a generalized integral identity involving $(p, q)$-differentiable functions.

The main idea behind the study of this paper is to obtain a new general post quantum integral inequality using twice $(p, q)$-differentiable functions. We then establish some new estimates of post quantum bounds essentially using the class of preinvex functions. We hope that the ideas and techniques of this paper will inspire interested readers working in this field.

Before we move to our next section of the paper, let us recall the definitions of invex set and preinvex function.

Definition 1.1 ([24]) Let $\mathcal{K} \subseteq \mathbb{R}$ be a nonempty set such that $a \in \mathcal{K}, 0<q<p \leq 1$, and let $f: \mathcal{K} \rightarrow \mathbb{R}$ be a continuous function. Then the $(p, q)$-derivative ${ }_{a} \mathcal{D}_{p, q} f(x)$ of $f$ at $x \in \mathcal{K}$ is defined by

$$
{ }_{a} \mathcal{D}_{p, q} f(x)=\frac{f(p x+(1-p) a)-f(q x+(1-q) a)}{(p-q)(x-a)} \quad(x \neq a) .
$$

Definition 1.2 ([24]) Let $\mathcal{K} \subseteq \mathbb{R}$ be a nonempty set such that $a \in \mathcal{K}, 0<q<p \leq 1$, and let $f: \mathcal{K} \rightarrow \mathbb{R}$ be a continuous function. Then $(p, q)$-integral on $\mathcal{K}$ is defined by

$$
\int_{a}^{x} f(t)_{a} \mathrm{~d}_{p, q} t=(p-q)(x-a) \sum_{n=0}^{\infty} \frac{q^{n}}{p^{n+1}} f\left(\frac{q^{n}}{p^{n+1}} x+\left(1-\frac{q^{n}}{p^{n+1}}\right) a\right)
$$

for $x \in \mathcal{K}$.

Definition 1.3 ([5]) A nonempty set $\mathcal{K} \subseteq \mathbb{R}$ is said to be invex with respect to the bivariate function $\zeta: \mathbb{R} \times \mathbb{R} \rightarrow \mathbb{R}$ if

$$
a+\mu \zeta(b, a) \in \mathcal{K}
$$

for all $a, b \in \mathcal{K}$ and $\mu \in[0,1]$. 
Definition 1.4 ([25]) Let $\mathcal{K} \subseteq \mathbb{R}$ be an invex set with respect to the bivariate function $\zeta: \mathbb{R} \times \mathbb{R} \rightarrow \mathbb{R}$. Then the real-valued function $f: \mathcal{K} \rightarrow \mathbb{R}$ is said to be preinvex with respect to $\zeta$ if

$$
f(a+\mu \zeta(b, a)) \leq(1-t) f(a)+t f(b)
$$

for all $a, b \in \mathcal{K}$ and $t \in[0,1]$.

\section{Results and discussions}

In this section, we derive our main results. First of all we derive our new post quantum integral identity involving twice $(p, q)$-differentiable function.

Lemma 2.1 Let $0<q<p \leq 1, f: \mathcal{K} \rightarrow \mathbb{R}$ be a twice $(p, q)$-differentiable function on $\mathcal{K}^{\circ}$ (where $\mathcal{K}^{\circ}$ is the interior of $\mathcal{K}$ ), and ${ }_{a} \mathcal{D}_{p, q}^{2}$ be continuous and $(p, q)$-integrable on $\mathcal{K}$. Then

$$
\begin{gathered}
\frac{q f(a)+p f(a+p \zeta(b, a))}{p+q}-\frac{1}{p^{2} \zeta(b, a)} \int_{a}^{a+p^{2} \zeta(b, a)} f(x)_{0} \mathrm{~d}_{p, q} x \\
=\frac{p q^{2} \zeta^{2}(b, a)}{p+q} \int_{0}^{1} t(1-q t)_{a} \mathcal{D}_{p, q}^{2} f(a+t \zeta(b, a))_{a} \mathrm{~d}_{p, q} t .
\end{gathered}
$$

Proof It suffices to prove that

$$
\begin{aligned}
{ }_{a} \mathcal{D}_{p, q}^{2} f(a+t \zeta(b, a)) \\
={ }_{a} \mathcal{D}_{p, q}\left(a \mathcal{D}_{p, q} f(a+t \zeta(b, a))\right) \\
=\frac{{ }_{a} \mathcal{D}_{p, q} f(a+p t \zeta(b, a))-{ }_{a} \mathcal{D}_{p, q} f(a+q t \zeta(b, a))}{t(p-q) \zeta(b, a)} \\
=\frac{1}{t(p-q) \zeta(b, a)}\left[\frac{f\left(a+p^{2} t \zeta(b, a)\right)-f(a+p q t \zeta(b, a))}{t p(p-q) \zeta(b, a)}\right. \\
\left.\quad-\frac{f(a+p q t \zeta(b, a))-f\left(a+q^{2} t \zeta(b, a)\right)}{t q(p-q) \zeta(b, a)}\right] \\
=\frac{q f\left(a+p^{2} t \zeta(b, a)\right)-(p+q) f(a+p q t \zeta(b, a))+p f\left(a+q^{2} t \zeta(b, a)\right)}{p q t^{2}(p-q)^{2} \zeta^{2}(b, a)} .
\end{aligned}
$$

Elaborated computation leads to

$$
\begin{array}{rl}
\int_{0}^{1} & t(1-q t)_{a} \mathcal{D}_{p, q}^{2} f(a+t \zeta(b, a))_{0} \mathrm{~d}_{p, q} t \\
= & \int_{0}^{1} t(1-q t) \\
& \times \frac{q f\left(a+p^{2} t \zeta(b, a)\right)-(p+q) f(a+p q t \zeta(b, a))+p f\left(a+q^{2} t \zeta(b, a)\right)}{t^{2} p q(p-q)^{2} \zeta^{2}(b, a)}{ }_{0} \mathrm{~d}_{p, q} t \\
= & \frac{1}{p q(p-q) \zeta^{2}(b, a)}\left[q \sum_{n=0}^{\infty} f\left(a+p^{2} \frac{q^{n}}{p^{n+1}} \zeta(b, a)\right)\right. \\
& \left.\quad-(p+q) \sum_{n=0}^{\infty} f\left(a+p \frac{q^{n+1}}{p^{n+1}} \zeta(b, a)\right)+p \sum_{n=0}^{\infty} f\left(a+\frac{q^{n+2}}{p^{n+1}} \zeta(b, a)\right)\right]
\end{array}
$$




$$
\begin{aligned}
& -q\left\{\frac{q(p-q) \zeta(b, a) \sum_{n=0}^{\infty} \frac{q^{n}}{p^{n+1}} f\left(a+p^{2} \frac{q^{n}}{p^{n+1}} \zeta(b, a)\right)}{p q(p-q)^{2} \zeta^{3}(b, a)}\right. \\
& -\frac{(p+q)(p-q) \zeta(b, a) \sum_{n=0}^{\infty} \frac{q^{n+1}}{p^{n+1}} f\left(a+p \frac{q^{n+1}}{p^{n+1}} \zeta(b, a)\right)}{p q^{2}(p-q)^{2} \zeta^{3}(b, a)} \\
& \left.+\frac{p(p-q) \zeta(b, a) \sum_{n=0}^{\infty} \frac{q^{n+2}}{p^{n+1}} f\left(a+\frac{q^{n+2}}{p^{n+1}} \zeta(b, a)\right)}{p q^{3}(p-q)^{2} \zeta^{3}(b, a)}\right\} \\
& =\frac{q\left[\sum_{n=0}^{\infty} f\left(a+p^{2} \frac{q^{n}}{p^{n+1}} \zeta(b, a)\right)-\sum_{n=0}^{\infty} f\left(a+p \frac{q^{n+1}}{p^{n+1}} \zeta(b, a)\right)\right]}{p q(p-q) \zeta^{2}(b, a)} \\
& -\frac{p\left[\sum_{n=0}^{\infty} f\left(a+p \frac{q^{n+1}}{p^{n+1}} \zeta(b, a)\right)-\sum_{n=0}^{\infty} f\left(a+\frac{q^{n+2}}{p^{n+1}} \zeta(b, a)\right)\right]}{p q(p-q) \zeta^{2}(b, a)} \\
& -q\left\{\frac{q(p-q) \zeta(b, a) \sum_{n=0}^{\infty} \frac{q^{n}}{p^{n+1}} f\left(a+p^{2} \frac{q^{n}}{p^{n+1}} \zeta(b, a)\right)}{p q(p-q)^{2} \zeta^{3}(b, a)}\right. \\
& -\frac{p(p+q)(p-q) \zeta(b, a) \sum_{n=0}^{\infty} \frac{q^{n+1}}{p^{n+2}} f\left(a+p^{2} \frac{q^{n+1}}{p^{n+2}} \zeta(b, a)\right)}{p q^{2}(p-q)^{2} \zeta^{3}(b, a)} \\
& \left.+\frac{p^{3}(p-q) \zeta(b, a) \sum_{n=0}^{\infty} \frac{q^{n+2}}{p^{n+3}} f\left(a+p^{2} \frac{q^{n+2}}{p^{n+3}} \zeta(b, a)\right)}{p q^{3}(p-q)^{2} \zeta^{3}(b, a)}\right\} \\
& =\frac{q[f(a+p \zeta(b, a))-f(a)]-p[f(a+q \zeta(b, a))-f(a)]}{p q(p-q) \zeta^{2}(b, a)} \\
& -\frac{p+q}{p^{3} q^{2} \zeta^{3}(b, a)} \int_{a}^{a+p^{2} \zeta(b, a)} f(x)_{0} \mathrm{~d}_{p, q} t \\
& -\frac{q^{2}+p q-p^{2}}{p q^{2}(p-q) \zeta^{2}(b, a)} f(a+p \zeta(b, a))+\frac{f(a+q \zeta(b, a))}{q(p-q) \zeta^{2}(b, a)} \\
& =\frac{f(a)}{p q \zeta^{2}(b, a)}+\frac{f(a+p \zeta(b, a))}{q^{2} \zeta^{2}(b, a)} \\
& -\frac{p+q}{p^{3} q^{2} \zeta^{3}(b, a)} \int_{a}^{a+p^{2} \zeta(b, a)} f(x)_{a} \mathrm{~d}_{p, q} x .
\end{aligned}
$$

Multiplying both sides of the above equality by $\frac{p q^{2} \zeta^{2}(b, a)}{p+q}$, we get the required result.

Theorem 2.2 Let $0<q<p \leq 1, \mathcal{K} \subseteq \mathbb{R}$ be an invex set with respect to the bivariate function $\zeta: \mathbb{R} \times \mathbb{R} \rightarrow \mathbb{R}$, and $f: \mathcal{K} \rightarrow \mathbb{R}$ be a twice $(p, q)$-differentiable function on $\mathcal{K}^{\circ}$ such that ${ }_{a} \mathcal{D}_{p, q}^{2} f$ is continuous and $(p, q)$-integrable on $\mathcal{K}$. Then the inequality

$$
\begin{aligned}
& \left|\frac{q f(a)+p f(a+p \zeta(b, a))}{p+q}-\frac{1}{p^{2} \zeta(b, a)} \int_{a}^{a+p^{2} \zeta(b, a)} f(x){ }_{a} \mathrm{~d}_{p, q} x\right| \\
& \quad \leq \frac{p q^{2} \zeta^{2}(b, a)\left(\left(p^{4}-p^{3}+p^{2} q^{2}\right)\left|{ }_{a} \mathcal{D}_{p, q}^{2} f(a)\right|+p^{3}\left|{ }_{a} \mathcal{D}_{p, q}^{2} f(b)\right|\right)}{(p+q)^{2}\left(p^{2}+q^{2}\right)\left(q^{2}+p q+p^{2}\right)}
\end{aligned}
$$

holds for all $a, b \in \mathcal{K}$ if $\left|{ }_{a} \mathcal{D}_{p, q}^{2} f\right|$ is a preinvex function with respect to $\zeta$. 
Proof It follows from Lemma 2.1 and the property of the modulus together with the preinvexity of $\left|{ }_{a} \mathcal{D}_{p, q}^{2}\right|$ that

$$
\begin{aligned}
& \left|\frac{q f(a)+p f(a+p \zeta(b, a))}{p+q}-\frac{1}{p^{2} \zeta(b, a)} \int_{a}^{a+p^{2} \zeta(b, a)} f(x)_{a} \mathrm{~d}_{p, q} x\right| \\
& \leq \frac{p q^{2} \zeta^{2}(b, a)}{p+q} \int_{0}^{1} t(1-q t)\left|{ }_{a} \mathcal{D}_{p, q}^{2} f(a+t \zeta(b, a))\right|{ }_{0} \mathrm{~d}_{p, q} t \\
& \quad \leq \frac{p q^{2} \zeta^{2}(b, a)}{p+q}\left(\left|{ }_{a} \mathcal{D}_{p, q}^{2} f(a)\right| \int_{0}^{1} t(1-t)(1-q t)_{0} \mathrm{~d}_{p, q} t\right. \\
& \left.\quad+\left|{ }_{a} \mathcal{D}_{p, q}^{2} f(b)\right| \int_{0}^{1} t^{2}(1-q t){ }_{0} \mathrm{~d}_{p, q} t\right) \\
& =\frac{p q^{2} \zeta^{2}(b, a)\left(\left.\left(p^{4}-p^{3}+p^{2} q^{2}\right)\right|_{a} \mathcal{D}_{p, q}^{2} f(a)\left|+p^{3}\right|_{a} \mathcal{D}_{p, q}^{2} f(b) \mid\right)}{(p+q)^{2}\left(p^{2}+q^{2}\right)\left(q^{2}+p q+p^{2}\right)}
\end{aligned}
$$

Theorem 2.3 Let $0<q<p \leq 1, r>1, \mathcal{K} \subseteq \mathbb{R}$ be an invex set with respect to the bivariate function $\zeta: \mathbb{R} \times \mathbb{R} \rightarrow \mathbb{R}$, and $f: \mathcal{K} \rightarrow \mathbb{R}$ be a twice $(p, q)$-differentiable function on $\mathcal{K}^{\circ}$ such that ${ }_{a} \mathcal{D}_{p, q}^{2} f$ is continuous and $(p, q)$-integrable on $\mathcal{K}$. Then the inequality

$$
\begin{aligned}
& \left|\frac{q f(a)+p f(a+p \zeta(b, a))}{p+q}-\frac{1}{p^{2} \zeta(b, a)} \int_{a}^{a+p^{2} \zeta(b, a)} f(x)_{a} \mathrm{~d}_{p, q} x\right| \\
& \quad \leq \frac{p q^{2} \zeta^{2}(b, a)}{(p+q)^{2-\frac{1}{r}}}\left(\left.\left.d_{1}\right|_{a} \mathcal{D}_{p, q}^{2} f(a)\right|^{r}+d_{2}\left|{ }_{a} \mathcal{D}_{p, q}^{2} f(b)\right|^{r}\right)^{\frac{1}{r}}
\end{aligned}
$$

is valid for all $a, b \in \mathcal{K}$ if $\left.\left.\right|_{a} \mathcal{D}_{p, q}^{2} f\right|^{r}$ is preinvex with respect to $\zeta$, where

$$
d_{1}=(p-q) \sum_{n=0}^{\infty}\left(\frac{q^{2 n}}{p^{2 n+2}}-\frac{q^{3 n}}{p^{3 n+3}}\right)\left(1-\frac{q^{n+1}}{p^{n+1}}\right)^{r}
$$

and

$$
d_{2}=(p-q) \sum_{n=0}^{\infty} \frac{q^{3 n}}{p^{3 n+3}}\left(1-\frac{q^{n+1}}{p^{n+1}}\right)^{r} .
$$

Proof From Lemma 2.1, Hölder's inequality, and the preinvexity of $\left|{ }_{a} \mathcal{D}_{p, q}^{2} f\right|^{r}$, we get

$$
\begin{aligned}
& \left|\frac{q f(a)+p f(a+p \zeta(b, a))}{p+q}-\frac{1}{p^{2} \zeta(b, a)} \int_{a}^{a+p^{2} \zeta(b, a)} f(x)_{a} \mathrm{~d}_{p, q} x\right| \\
& \quad \leq\left.\left.\frac{p q^{2} \zeta^{2}(b, a)}{p+q} \int_{0}^{1} t(1-q t)\right|_{a} \mathcal{D}_{p, q}^{2} f(a+t \zeta(b, a))\right|_{0} \mathrm{~d}_{p, q} t \\
& \quad \leq \frac{p q^{2} \zeta^{2}(b, a)}{p+q}\left(\int_{0}^{1} t_{0} \mathrm{~d}_{p, q} t\right)^{1-\frac{1}{r}}\left(\left.\left.\int_{0}^{1} t(1-q t)^{r}\right|_{a} \mathcal{D}_{p, q}^{2} f(a+t \zeta(b, a))\right|^{r} \mathrm{~d}_{p, q} t\right)^{\frac{1}{r}} \\
& \quad \leq \frac{p q^{2} \zeta^{2}(b, a)}{p+q}\left(\frac{1}{p+q}\right)^{1-\frac{1}{r}}
\end{aligned}
$$




$$
\begin{aligned}
& \times\left(\left|{ }_{a} \mathcal{D}_{p, q}^{2} f(a)\right|^{r} \int_{0}^{1} t(1-t)(1-q t)^{r}{ }_{0} \mathrm{~d}_{p, q} t+\left|{ }_{a} \mathcal{D}_{p, q}^{2} f(b)\right|^{r} \int_{0}^{1} t^{2}(1-q t)^{r}{ }_{0} \mathrm{~d}_{p, q} t\right)^{\frac{1}{r}} \\
= & \frac{p q^{2} \zeta^{2}(b, a)}{(p+q)^{2-\frac{1}{r}}}\left(d_{1}\left|{ }_{a} \mathcal{D}_{p, q}^{2} f(a)\right|^{r}+d_{2}\left|{ }_{a} \mathcal{D}_{p, q}^{2} f(b)\right|^{r}\right)^{\frac{1}{r}}
\end{aligned}
$$

Theorem 2.4 Let $0<q<p \leq 1, r, s>1$ with $1 / r+1 / s=1, \mathcal{K} \subseteq \mathbb{R}$ be an invex set with respect to the bivariate function $\zeta: \mathbb{R} \times \mathbb{R} \rightarrow \mathbb{R}$, and $f: \mathcal{K} \rightarrow \mathbb{R}$ be a twice $(p, q)$-differentiable function on $\mathcal{K}^{\circ}$ such that ${ }_{a} \mathcal{D}_{p, q}^{2} f$ is continuous and $(p, q)$-integrable on $\mathcal{K}$. Then the inequality

$$
\begin{aligned}
& \left|\frac{q f(a)+p f(a+p \zeta(b, a))}{p+q}-\frac{1}{p^{2} \zeta(b, a)} \int_{a}^{a+p^{2} \zeta(b, a)} f(x){ }_{a} \mathrm{~d}_{p, q} x\right| \\
& \quad \leq \frac{p q^{2} \zeta^{2}(b, a)}{(p+q)} h^{\frac{1}{s}}\left(\frac{\left(q^{2}+p^{2}+p q-p-q\right)\left|{ }_{a} \mathcal{D}_{p, q}^{2} f(a)\right|^{r}+(p+q)\left|{ }_{a} \mathcal{D}_{p, q}^{2} f(b)\right|^{r}}{(p+q)\left(q^{2}+p q+p^{2}\right)}\right)^{\frac{1}{r}}
\end{aligned}
$$

takes place for all $a, b \in \mathcal{K}$ if $\left|{ }_{a} \mathcal{D}_{p, q}^{2}\right|^{r}$ is preinvex with respect to $\zeta$, where

$$
h=(p-q) \sum_{n=0}^{\infty} \frac{q^{2 n}}{p^{2 n+2}}\left(1-\frac{q^{n}}{p^{n+1}}\right)^{s} .
$$

Proof Using Lemma 2.1, Hölder's inequality, and the preinvexity of $\left|{ }_{a} \mathcal{D}_{p, q}^{2} f\right|^{r}$, we have

$$
\begin{aligned}
& \left|\frac{q f(a)+p f(a+p \zeta(b, a))}{p+q}-\frac{1}{p^{2} \zeta(b, a)} \int_{a}^{a+p^{2} \zeta(b, a)} f(x)_{a} \mathrm{~d}_{p, q} x\right| \\
& \leq\left.\left.\frac{p q^{2} \zeta^{2}(b, a)}{p+q} \int_{0}^{1} t(1-q t)\right|_{a} \mathcal{D}_{p, q}^{2} f(a+t \zeta(b, a))\right|_{0} \mathrm{~d}_{p, q} t \\
& \leq \frac{p q^{2} \zeta^{2}(b, a)}{p+q}\left(\int_{0}^{1} t(1-q t)^{s}{ }_{0} \mathrm{~d}_{p, q} t\right)\left(\left.\left.\int_{0}^{1} t\right|_{a} \mathcal{D}_{p, q}^{2} f(a+t \zeta(b, a))\right|^{r}{ }_{0} \mathrm{~d}_{p, q} t\right)^{\frac{1}{r}} \\
& \leq \frac{p q^{2} \zeta^{2}(b, a)}{p+q} \\
& \quad \times\left(\int_{0}^{1} t(1-q t)^{s}{ }_{0} \mathrm{~d}_{p, q} t\right)^{\frac{1}{s}} \\
& \quad \times\left(\left|{ }_{a} \mathcal{D}_{p, q}^{2} f(a)\right|^{r} \int_{0}^{1} t(1-t)_{0} \mathrm{~d}_{p, q} t+\left|{ }_{a} \mathcal{D}_{p, q}^{2} f(b)\right|^{r} \int_{0}^{1} t^{2}{ }_{0} \mathrm{~d}_{p, q} t\right)^{\frac{1}{r}} \\
& =\frac{p q^{2} \zeta^{2}(b, a)}{(p+q)} h^{\frac{1}{s}}\left(\frac{\left(q^{2}+p^{2}+p q-p-q\right)\left|{ }_{a} \mathcal{D}_{p, q}^{2} f(a)\right|^{r}+(p+q)\left|{ }_{a} \mathcal{D}_{p, q}^{2} f(b)\right|^{r}}{(p+q)\left(q^{2}+p q+p^{2}\right)}\right)^{\frac{1}{r}} .
\end{aligned}
$$

Theorem 2.5 Let $0<q<p \leq 1, r>1, \mathcal{K} \subseteq \mathbb{R}$ be an invex set with respect to the bivariate function $\zeta: \mathbb{R} \times \mathbb{R} \rightarrow \mathbb{R}$, and $f: \mathcal{K} \rightarrow \mathbb{R}$ be a twice $(p, q)$-differentiable function on $\mathcal{K}^{\circ}$ such that ${ }_{a} \mathcal{D}_{p, q}^{2} f$ is continuous and $(p, q)$-integrable on $\mathcal{K}$. Then one has

$$
\begin{aligned}
& \left|\frac{q f(a)+p f(a+p \zeta(b, a))}{p+q}-\frac{1}{p^{2} \zeta(b, a)} \int_{a}^{a+p^{2} \zeta(b, a)} f(x)_{a} \mathrm{~d}_{p, q} x\right| \\
& \quad \leq \frac{p q^{2} \zeta^{2}(b, a)}{(p+q)}\left(k_{1}\left|{ }_{a} \mathcal{D}_{p, q}^{2} f(a)\right|^{r}+k_{2}\left|{ }_{a} \mathcal{D}_{p, q}^{2} f(b)\right|^{r}\right)^{\frac{1}{r}}
\end{aligned}
$$


for all $a, b \in \mathcal{K}$ if $\left.\left.\right|_{a} \mathcal{D}_{p, q}^{2} f\right|^{r}$ is a preinvex function with respect to $\zeta$, where

$$
k_{1}=(p-q) \sum_{n=0}^{\infty}\left(\frac{q^{n}}{p^{n+1}}\right)^{r+1}\left(1-\frac{q^{n}}{p^{n+1}}\right)\left(1-\frac{q^{n+1}}{p^{n+1}}\right)^{r}
$$

and

$$
k_{2}=(p-q) \sum_{n=0}^{\infty}\left(\frac{q^{n}}{p^{n+1}}\right)^{r+3}\left(1-\frac{q^{n+1}}{p^{n+1}}\right)^{r} .
$$

Proof It follows from Lemma 2.1 and Hölder's inequality together with the preinvexity of $\left|{ }_{a} \mathcal{D}_{p, q}^{2} f\right|^{r}$ that

$$
\begin{aligned}
& \left|\frac{q f(a)+p f(a+p \zeta(b, a))}{p+q}-\frac{1}{p^{2} \zeta(b, a)} \int_{a}^{a+p^{2} \zeta(b, a)} f(x)_{a} \mathrm{~d}_{p, q} x\right| \\
& \leq\left.\frac{p q^{2} \zeta^{2}(b, a)}{p+q} \int_{0}^{1} t(1-q t)\right|_{a} \mathcal{D}_{p, q}^{2} f(a+t \zeta(b, a)) \mid{ }_{0} \mathrm{~d}_{p, q} t \\
& \leq \frac{p q^{2} \zeta^{2}(b, a)}{p+q}\left(\int_{0}^{1} 1_{0} \mathrm{~d}_{p, q} t\right)^{1-\frac{1}{r}}\left(\int_{0}^{1} t^{r}(1-q t)^{r}\left|{ }_{a} \mathcal{D}_{p, q}^{2} f(a+t \zeta(b, a))\right|^{r}{ }_{0} \mathrm{~d}_{p, q} t\right)^{\frac{1}{r}} \\
& \leq \frac{p q^{2} \zeta^{2}(b, a)}{p+q} \\
& \quad \times\left(\left|{ }_{a} \mathcal{D}_{p, q}^{2} f(a)\right|^{r} \int_{0}^{1} t^{r}(1-t)(1-q t)^{r}{ }_{0} \mathrm{~d}_{p, q} t+\left|{ }_{a} \mathcal{D}_{p, q}^{2} f(b)\right|^{r}\right. \\
& \left.\quad \times \int_{0}^{1} t^{r+2}(1-q t)^{r}{ }_{0} \mathrm{~d}_{p, q} t\right)^{\frac{1}{r}} \\
& =\frac{p q^{2} \zeta^{2}(b, a)}{(p+q)}\left(k_{1}\left|{ }_{a} \mathcal{D}_{p, q}^{2} f(a)\right|^{r}+k_{2}\left|{ }_{a} \mathcal{D}_{p, q}^{2} f(b)\right|^{r}\right)^{\frac{1}{r}} .
\end{aligned}
$$

Theorem 2.6 Let $0<q<p \leq 1, r, s>1$ with $1 / r+1 / s=1, \mathcal{K} \subseteq \mathbb{R}$ be an invex set with respect to the bivariate function $\zeta: \mathbb{R} \times \mathbb{R} \rightarrow \mathbb{R}$, and $f: \mathcal{K} \rightarrow \mathbb{R}$ be a twice $(p, q)$-differentiable function on $\mathcal{K}^{\circ}$ such that ${ }_{a} \mathcal{D}_{p, q}^{2} f$ is continuous and $(p, q)$-integrable on $\mathcal{K}$. Then the inequality

$$
\begin{gathered}
\left|\frac{q f(a)+p f(a+p \zeta(b, a))}{p+q}-\frac{1}{p^{2} \zeta(b, a)} \int_{a}^{a+p^{2} \zeta(b, a)} f(x)_{a} \mathrm{~d}_{p, q} x\right| \\
\leq \frac{p q^{2} \zeta^{2}(b, a)}{(p+q)} m^{\frac{1}{s}}\left(\frac{\left.\left.q\right|_{a} \mathcal{D}_{p, q}^{2} f(a)\right|^{r}+\left.{ }_{a} \mathcal{D}_{p, q}^{2} f(b)\right|^{r}}{(1+q)}\right)^{\frac{1}{r}}
\end{gathered}
$$

holds for all $a, b \in \mathcal{K}$ if $\left|{ }_{a} \mathcal{D}_{p, q}^{2} f\right|^{r}$ is preinvex with respect to $\zeta$, where

$$
m=(p-q) \sum_{n=0}^{\infty}\left(\frac{q^{n}}{p^{n+1}}\right)^{s+1}\left(1-\frac{q^{n+1}}{p^{n+1}}\right)^{s} .
$$


Proof Making use of Lemma 2.1, Hölder's inequality, and the preinvexity of $\left|{ }_{a} \mathcal{D}_{p, q}^{2} f\right|^{r}$, we have

$$
\begin{aligned}
& \left|\frac{q f(a)+p f(a+p \zeta(b, a))}{p+q}-\frac{1}{p^{2} \zeta(b, a)} \int_{a}^{a+p^{2} \zeta(b, a)} f(x)_{a} \mathrm{~d}_{p, q} x\right| \\
& \quad \leq\left.\left.\frac{p q^{2} \zeta^{2}(b, a)}{p+q} \int_{0}^{1} t(1-q t)\right|_{a} \mathcal{D}_{p, q}^{2} f(a+t \zeta(b, a))\right|_{0} \mathrm{~d}_{p, q} t \\
& \leq \frac{p q^{2} \zeta^{2}(b, a)}{p+q}\left(\int_{0}^{1} t^{s}(1-q t)_{0}^{s} \mathrm{~d}_{p, q} t\right)^{\frac{1}{s}}\left(\int_{0}^{1}\left|{ }_{a} \mathcal{D}_{p, q}^{2} f(a+t \zeta(b, a))\right|^{r}{ }_{0} \mathrm{~d}_{p, q} t\right)^{\frac{1}{r}} \\
& \leq \frac{p q^{2} \zeta^{2}(b, a)}{p+q}\left(\int_{0}^{1} t^{s}(1-q t)^{s}{ }_{0} \mathrm{~d}_{p, q} t\right)^{\frac{1}{s}} \\
& \quad \times\left(\left|{ }_{a} \mathcal{D}_{p, q}^{2} f(a)\right|^{r} \int_{0}^{1}(1-t)_{0} \mathrm{~d}_{p, q} t+\left|{ }_{a} \mathcal{D}_{p, q}^{2} f(b)\right|^{r} \int_{0}^{1} t_{0} \mathrm{~d}_{p, q} t\right)^{\frac{1}{r}} \\
& =\frac{p q^{2} \zeta^{2}(b, a)}{(p+q)} m^{\frac{1}{s}}\left(\frac{\left.\left.(p+q-1)\right|_{a} \mathcal{D}_{p, q}^{2} f(a)\right|^{r}+\left.{ }_{a} \mathcal{D}_{p, q}^{2} f(b)\right|^{r}}{(p+q)}\right)^{\frac{1}{r}} .
\end{aligned}
$$

Theorem 2.7 Let $0<q<p \leq 1, r, s>1$ with $1 / r+1 / s=1, \mathcal{K} \subseteq \mathbb{R}$ be an invex set with respect to the bivariate function $\zeta: \mathbb{R} \times \mathbb{R} \rightarrow \mathbb{R}$, and $f: \mathcal{K} \rightarrow \mathbb{R}$ be a twice $(p, q)$-differentiable function on $\mathcal{K}^{\circ}$ such that ${ }_{a} \mathcal{D}_{p, q}^{2} f$ is continuous and $(p, q)$-integrable on $\mathcal{K}$. Then

$$
\begin{aligned}
& \left|\frac{q f(a)+p f(a+p \zeta(b, a))}{p+q}-\frac{1}{p^{2} \zeta(b, a)} \int_{a}^{a+p^{2} \zeta(b, a)} f(x)_{a} \mathrm{~d}_{p, q} x\right| \\
& \quad \leq \frac{p q^{2} \zeta^{2}(b, a)}{(p+q)}\left(\frac{p-q}{p^{s+1}-q^{s+1}}\right)^{\frac{1}{s}}\left(u_{1}\left|{ }_{a} \mathcal{D}_{p, q}^{2} f(a)\right|^{r}+u_{2}\left|{ }_{a} \mathcal{D}_{p, q}^{2} f(b)\right|^{r}\right)^{\frac{1}{r}}
\end{aligned}
$$

for all $a, b \in \mathcal{K}$ if $\left|{ }_{a} \mathcal{D}_{p, q}^{2} f\right|^{r}$ is a preinvex function with respect to $\zeta$, where

$$
u_{1}=(p-q) \sum_{n=0}^{\infty}\left(\frac{q^{n}}{p^{n+1}}-\frac{q^{2 n}}{p^{2 n+2}}\right)\left(1-\frac{q^{n+1}}{p^{n+1}}\right)^{r}
$$

and

$$
u_{2}=(p-q) \sum_{n=0}^{\infty} \frac{q^{2 n}}{p^{2 n+2}}\left(1-\frac{q^{n+1}}{p^{n+1}}\right)^{r}
$$

Proof From Lemma 2.1, Hölder's inequality, and the preinvexity of $\left.\left.\right|_{a} \mathcal{D}_{p, q}^{2} f\right|^{r}$, we have

$$
\begin{aligned}
& \left|\frac{q f(a)+p f(a+p \zeta(b, a))}{p+q}-\frac{1}{p^{2} \zeta(b, a)} \int_{a}^{a+p^{2} \zeta(b, a)} f(x)_{a} \mathrm{~d}_{p, q} x\right| \\
& \quad \leq\left.\left.\frac{p q^{2} \zeta^{2}(b, a)}{p+q} \int_{0}^{1} t(1-q t)\right|_{a} \mathcal{D}_{p, q}^{2} f(a+t \zeta(b, a))\right|_{0} \mathrm{~d}_{p, q} t \\
& \quad \leq \frac{p q^{2} \zeta^{2}(b, a)}{p+q}\left(\int_{0}^{1} t^{s}{ }_{0} \mathrm{~d}_{p, q} t\right)^{\frac{1}{s}}\left(\left.\left.\int_{0}^{1}(1-q t)^{r}\right|_{a} \mathcal{D}_{p, q}^{2} f(a+t \zeta(b, a))\right|_{0} ^{r} \mathrm{~d}_{p, q} t\right)^{\frac{1}{r}} \\
& \quad \leq \frac{p q^{2} \zeta^{2}(b, a)}{p+q}\left(\frac{p-q}{p^{s+1}-q^{s+1}}\right)^{\frac{1}{s}}
\end{aligned}
$$




$$
\begin{aligned}
& \times\left(\left|{ }_{a} \mathcal{D}_{p, q}^{2} f(a)\right|^{r} \int_{0}^{1}(1-t)(1-q t)^{r}{ }_{0} \mathrm{~d}_{p, q} t+\left|{ }_{a} \mathcal{D}_{p, q}^{2} f(b)\right|^{r} \int_{0}^{1} t(1-q t)^{r}{ }_{0} \mathrm{~d}_{p, q} t\right)^{\frac{1}{r}} \\
= & \frac{p q^{2} \zeta^{2}(b, a)}{(p+q)}\left(\frac{p-q}{p^{s+1}-q^{s+1}}\right)^{\frac{1}{s}}\left(u_{1}\left|{ }_{a} \mathcal{D}_{p, q}^{2} f(a)\right|^{r}+u_{2}\left|{ }_{a} \mathcal{D}_{p, q}^{2} f(b)\right|^{r}\right)^{\frac{1}{r}} .
\end{aligned}
$$

Theorem 2.8 Let $0<q<p \leq 1, r, s>1$ with $1 / r+1 / s=1, \mathcal{K} \subseteq \mathbb{R}$ be an invex set with respect to the bivariate function $\zeta: \mathbb{R} \times \mathbb{R} \rightarrow \mathbb{R}$, and $f: \mathcal{K} \rightarrow \mathbb{R}$ be a twice $(p, q)$-differentiable function on $\mathcal{K}^{\circ}$ such that ${ }_{a} \mathcal{D}_{p, q}^{2} f$ is continuous and $(p, q)$-integrable on $\mathcal{K}$. Then the inequality

$$
\begin{aligned}
& \left|\frac{q f(a)+p f(a+p \zeta(b, a))}{p+q}-\frac{1}{p^{2} \zeta(b, a)} \int_{a}^{a+p^{2} \zeta(b, a)} f(x)_{a} \mathrm{~d}_{p, q} x\right| \\
& \leq \frac{p q^{2} \zeta^{2}(b, a)}{(p+q)} w^{\frac{1}{s}}\left(\left(\frac{p-q}{p^{r+1}-q^{r+1}}-\frac{p-q}{p^{r+2}-q^{r+2}}\right)\left|{ }_{a} \mathcal{D}_{p, q}^{2} f(a)\right|^{r}\right. \\
& \left.\quad+\frac{p-q}{p^{r+2}-q^{r+2}}\left|{ }_{a} \mathcal{D}_{p, q}^{2} f(b)\right|^{r}\right)^{\frac{1}{r}}
\end{aligned}
$$

holds for all $a, b \in \mathcal{K}$ if $\left|{ }_{a} \mathcal{D}_{p, q}^{2} f\right|^{r}$ is a preinvex function with respect to $\zeta$, where

$$
w=(p-q) \sum_{n=0}^{\infty} \frac{q^{n}}{p^{n+1}}\left(1-\frac{q^{n+1}}{p^{n+1}}\right)^{s} .
$$

Proof According to Lemma 2.1, Hölder's inequality, and the preinvexity of $\left|{ }_{a} \mathcal{D}_{p, q}^{2} f\right|^{r}$, one has

$$
\begin{aligned}
& \left|\frac{q f(a)+p f(a+p \zeta(b, a))}{p+q}-\frac{1}{p^{2} \zeta(b, a)} \int_{a}^{a+p^{2} \zeta(b, a)} f(x)_{a} \mathrm{~d}_{p, q} x\right| \\
& \leq\left.\frac{p q^{2} \zeta^{2}(b, a)}{p+q} \int_{0}^{1}(1-q t)\right|_{a} \mathcal{D}_{p, q}^{2} f(a+t \zeta(b, a)) \mid{ }_{0} \mathrm{~d}_{p, q} t \\
& \leq \frac{p q^{2} \zeta^{2}(b, a)}{p+q}\left(\int_{0}^{1}(1-q t)_{0}^{s} \mathrm{~d}_{p, q} t\right)^{\frac{1}{s}}\left(\left.\left.\int_{0}^{1} t^{r}\right|_{a} \mathcal{D}_{p, q}^{2} f(a+t \zeta(b, a))\right|_{0} ^{r} \mathrm{~d}_{p, q}\right)^{\frac{1}{r}} \\
& \leq \frac{p q^{2} \zeta^{2}(b, a)}{p+q}\left(\int_{0}^{1}(1-q t)_{0}^{s} \mathrm{~d}_{p, q} t\right)^{\frac{1}{s}} \\
& \quad \times\left(\left|{ }_{a} \mathcal{D}_{p, q}^{2} f(a)\right|^{r} \int_{0}^{1} t^{r}(1-t)_{0} \mathrm{~d}_{p, q} t+\left|{ }_{a} \mathcal{D}_{p, q}^{2} f(b)\right|^{r} \int_{0}^{1} t^{r+1}{ }_{0} \mathrm{~d}_{p, q} t\right)^{\frac{1}{r}} \\
& =\frac{p q^{2} \zeta^{2}(b, a)}{(p+q)} w^{\frac{1}{s}}\left(\left(\frac{p-q}{p^{r+1}-q^{r+1}}-\frac{p-q}{p^{r+2}-q^{r+2}}\right)\left|{ }_{a} \mathcal{D}_{p, q}^{2} f(a)\right|^{r}\right. \\
& \left.\quad+\frac{p-q}{p^{r+2}-q^{r+2}}\left|{ }_{a} \mathcal{D}_{p, q}^{2} f(b)\right|^{r}\right)^{\frac{1}{r}} .
\end{aligned}
$$

Theorem 2.9 Let $0<q<p \leq 1, r>1, \mathcal{K} \subseteq \mathbb{R}$ be an invex set with respect to the bivariate function $\zeta: \mathbb{R} \times \mathbb{R} \rightarrow \mathbb{R}$, and $f: \mathcal{K} \rightarrow \mathbb{R}$ be a twice $(p, q)$-differentiable function on $\mathcal{K}^{\circ}$ such 
that ${ }_{a} \mathcal{D}_{p, q}^{2} f$ is continuous and $(p, q)$-integrable on $\mathcal{K}$. Then

$$
\begin{aligned}
& \left|\frac{q f(a)+p f(a+p \zeta(b, a))}{p+q}-\frac{1}{p^{2} \zeta(b, a)} \int_{a}^{a+p^{2} \zeta(b, a)} f(x)_{a} \mathrm{~d}_{p, q} x\right| \\
& \leq \frac{p^{2-\frac{1}{r}} q^{2} \zeta^{2}(b, a)}{(p+q)^{2-\frac{1}{r}}}\left(\frac{p^{2}}{q^{2}+p q+p^{2}}\right)^{1-\frac{1}{r}} \\
& \quad \times\left(\frac{\left(p^{4}-p^{3}+p^{2} q^{2}\right)\left|{ }_{a} \mathcal{D}_{p, q}^{2} f(a)\right|^{r}+p^{3}\left|{ }_{a} \mathcal{D}_{p, q}^{2} f(b)\right|^{r}}{(p+q)\left(p^{2}+q^{2}\right)\left(q^{2}+p q+p^{2}\right)}\right)^{\frac{1}{r}}
\end{aligned}
$$

for all $a, b \in \mathcal{K}$ if $\left|{ }_{a} \mathcal{D}_{p, q}^{2} f\right|^{r}$ is preinvex with respect to $\zeta$.

Proof Lemma 2.1, Hölder's inequality, and the preinvexity of $\left|{ }_{a} \mathcal{D}_{p, q}^{2} f\right|^{r}$ lead to

$$
\begin{aligned}
& \left|\frac{q f(a)+p f(a+p \zeta(b, a))}{p+q}-\frac{1}{p^{2} \zeta(b, a)} \int_{a}^{a+p^{2} \zeta(b, a)} f(x)_{a} \mathrm{~d}_{p, q} x\right| \\
& \leq\left.\left.\frac{p q^{2} \zeta^{2}(b, a)}{p+q} \int_{0}^{1} t(1-q t)\right|_{a} \mathcal{D}_{p, q}^{2} f(a+t \zeta(b, a))\right|_{0} \mathrm{~d}_{p, q} t \\
& \leq \frac{p q^{2} \zeta^{2}(b, a)}{p+q}\left(\int_{0}^{1} t(1-q t)_{0} \mathrm{~d}_{p, q} t\right)^{1-\frac{1}{r}} \\
& \quad \times\left(\left.\left.\int_{0}^{1} t(1-q t)\right|_{a} \mathcal{D}_{p, q}^{2} f(a+t \zeta(b, a))\right|^{r}{ }_{0} \mathrm{~d}_{p, q} t\right)^{\frac{1}{r}} \\
& \leq \frac{p q^{2} \zeta^{2}(b, a)}{p+q}\left(\int_{0}^{1} t(1-q t)_{0} \mathrm{~d}_{p, q} t\right)^{1-\frac{1}{r}} \\
& \quad \times\left(\left|{ }_{a} \mathcal{D}_{p, q}^{2} f(a)\right|^{r} \int_{0}^{1} t(1-t)(1-q t)_{0} \mathrm{~d}_{p, q} t+\left|{ }_{a} \mathcal{D}_{p, q}^{2} f(b)\right|^{r} \int_{0}^{1} t^{2}(1-q t)_{0} \mathrm{~d}_{p, q} t\right)^{\frac{1}{r}} \\
& =\frac{p^{2-\frac{1}{r}} q^{2} \zeta^{2}(b, a)}{(p+q)^{2-\frac{1}{r}}}\left(\frac{p^{2}}{q^{2}+p q+p^{2}}\right)^{1-\frac{1}{r}} \\
& \quad \times\left(\frac{\left.\left.\left(p^{4}-p^{3}+p^{2} q^{2}\right)\right|_{a} \mathcal{D}_{p, q}^{2} f(a)\right|^{r}+\left.\left.p^{3}\right|_{a} \mathcal{D}_{p, q}^{2} f(b)\right|^{r}}{(p+q)\left(p^{2}+q^{2}\right)\left(q^{2}+p q+p^{2}\right)}\right)^{\frac{1}{r}} .
\end{aligned}
$$

Theorem 2.10 Let $0<q<p \leq 1, r>1, \mathcal{K} \subseteq \mathbb{R}$ be an invex set with respect to the bivariate function $\zeta: \mathbb{R} \times \mathbb{R} \rightarrow \mathbb{R}$, and $f: \mathcal{K} \rightarrow \mathbb{R}$ be a twice $(p, q)$-differentiable function on $\mathcal{K}^{\circ}$ such that ${ }_{a} \mathcal{D}_{p, q}^{2} f$ is continuous and $(p, q)$-integrable on $\mathcal{K}$. Then one has

$$
\begin{aligned}
& \left|\frac{q f(a)+p f(a+p \zeta(b, a))}{p+q}-\frac{1}{p^{2} \zeta(b, a)} \int_{a}^{a+p^{2} \zeta(b, a)} f(x){ }_{a} \mathrm{~d}_{p, q} x\right| \\
& \leq \frac{p^{2-\frac{1}{r}} q^{2} \zeta^{2}(b, a)}{(p+q)^{2-\frac{1}{r}}}\left(\left[\frac{p-q}{p^{r+1}-q^{r+1}}-\frac{(p-q)(1+q)}{p^{r+2}-q^{r+2}}+\frac{q(p-q)}{p^{r+3}-q^{r+3}}\right]\left|{ }_{a} \mathcal{D}_{p, q}^{2} f(a)\right|^{r}\right. \\
& \left.\quad+\left[\frac{p-q}{p^{r+2}-q^{r+2}}-\frac{q(p-q)}{p^{r+3}-q^{r+3}}\right]\left|{ }_{a} \mathcal{D}_{p, q}^{2} f(b)\right|^{r}\right)^{\frac{1}{r}}
\end{aligned}
$$

for all $a, b \in \mathcal{K}$ if $\left|{ }_{a} \mathcal{D}_{p, q}^{2} f\right|^{r}$ is a preinvex function with respect to $\zeta$. 
Proof Making use of Lemma 2.1, Hölder's inequality, and the preinvexity of $\left|{ }_{a} \mathcal{D}_{p, q}^{2} f\right|^{r}$, we have

$$
\begin{aligned}
& \left|\frac{q f(a)+p f(a+p \zeta(b, a))}{p+q}-\frac{1}{p^{2} \zeta(b, a)} \int_{a}^{a+p^{2} \zeta(b, a)} f(x)_{a} \mathrm{~d}_{p, q} x\right| \\
& \leq\left.\left.\frac{p q^{2} \zeta^{2}(b, a)}{p+q} \int_{0}^{1} t(1-q t)\right|_{a} \mathcal{D}_{p, q}^{2} f(a+t \zeta(b, a))\right|_{0} \mathrm{~d}_{p, q} t \\
& \leq \frac{p q^{2} \zeta^{2}(b, a)}{p+q}\left(\int_{0}^{1}(1-q t)_{0} \mathrm{~d}_{p, q} t\right)^{1-\frac{1}{r}}\left(\left.\left.\int_{0}^{1} t^{r}(1-q t)\right|_{a} \mathcal{D}_{p, q}^{2} f(a+t \zeta(b, a))\right|_{0} ^{r} \mathrm{~d}_{p, q} t\right)^{\frac{1}{r}} \\
& \leq \frac{p q^{2} \zeta^{2}(b, a)}{p+q}\left(\int_{0}^{1}(1-q t)_{0} \mathrm{~d}_{p, q} t\right)^{1-\frac{1}{r}} \\
& \quad \times\left(\left|{ }_{a} \mathcal{D}_{p, q}^{2} f(a)\right|^{r} \int_{0}^{1} t^{r}(1-t)(1-q t)_{0} \mathrm{~d}_{p, q} t+\left|{ }_{a} \mathcal{D}_{p, q}^{2} f(b)\right|^{r} \int_{0}^{1} t^{r+1}(1-q t)_{0} \mathrm{~d}_{p, q} t\right)^{\frac{1}{r}} \\
& =\frac{p^{2-\frac{1}{r}} q^{2} \zeta^{2}(b, a)}{(p+q)^{2-\frac{1}{r}}}\left(\left[\frac{p-q}{p^{r+1}-q^{r+1}}-\frac{(p-q)(1+q)}{p^{r+2}-q^{r+2}}+\frac{q(p-q)}{p^{r+3}-q^{r+3}}\right]\left|{ }_{a} \mathcal{D}_{p, q}^{2} f(a)\right|^{r}\right. \\
& \left.\quad+\left[\frac{p-q}{p^{r+2}-q^{r+2}}-\frac{q(p-q)}{p^{r+3}-q^{r+3}}\right]\left|{ }_{a} \mathcal{D}_{p, q}^{2} f(b)\right|^{r}\right)^{\frac{1}{r}} .
\end{aligned}
$$

Theorem 2.11 Let $0<q<p \leq 1, r, s>1$ with $1 / r+1 / s=1, \mathcal{K} \subseteq \mathbb{R}$ be an invex set with respect to the bivariate function $\zeta: \mathbb{R} \times \mathbb{R} \rightarrow \mathbb{R}$, and $f: \mathcal{K} \rightarrow \mathbb{R}$ be a twice $(p, q)$-differentiable function on $\mathcal{K}^{\circ}$ such that ${ }_{a} \mathcal{D}_{p, q}^{2} f$ is continuous and $(p, q)$-integrable on $\mathcal{K}$. Then the inequality

$$
\begin{aligned}
& \left|\frac{q f(a)+p f(a+p \zeta(b, a))}{p+q}-\frac{1}{p^{2} \zeta(b, a)} \int_{a}^{a+p^{2} \zeta(b, a)} f(x){ }_{a} \mathrm{~d}_{p, q} x\right| \\
& \quad \leq \frac{p q^{2} \zeta^{2}(b, a)}{(p+q)} \lambda^{\frac{1}{s}}\left(\frac{\left(p^{3}-p^{2}+p q^{2}+p^{2} q\right)\left|{ }_{a} \mathcal{D}_{p, q}^{2} f(a)\right|^{r}+\left.\left.p^{2}\right|_{a} \mathcal{D}_{p, q}^{2} f(b)\right|^{r}}{(p+q)\left(q^{2}+p q+p^{2}\right)}\right)^{\frac{1}{r}}
\end{aligned}
$$

holds for all $a, b \in \mathcal{K}$ if $\left|{ }_{a} \mathcal{D}_{p, q}^{2} f\right|^{r}$ is preinvex function with respect to $\zeta$, where

$$
\lambda=\frac{p-q}{p^{s+1}-q^{s+1}}-\frac{q(p-q)}{p^{s+2}-q^{s+2}}
$$

Proof It follows from Lemma 2.1, Hölder's inequality, and the preinvexity of $\left|{ }_{a} \mathcal{D}_{p, q}^{2} f\right|^{r}$ that

$$
\begin{aligned}
& \left|\frac{q f(a)+p f(a+p \zeta(b, a))}{p+q}-\frac{1}{p^{2} \zeta(b, a)} \int_{a}^{a+p^{2} \zeta(b, a)} f(x)_{a} \mathrm{~d}_{p, q} x\right| \\
& \quad \leq\left.\left.\frac{p q^{2} \zeta^{2}(b, a)}{p+q} \int_{0}^{1} t(1-q t)\right|_{a} \mathcal{D}_{p, q}^{2} f(a+t \zeta(b, a))\right|_{0} \mathrm{~d}_{p, q} t \\
& \quad \leq \frac{p q^{2} \zeta^{2}(b, a)}{p+q}\left(\int_{0}^{1} t^{s}(1-q t)_{0} \mathrm{~d}_{p, q} t\right)^{\frac{1}{s}}\left(\left.\left.\int_{0}^{1}(1-q t)\right|_{a} \mathcal{D}_{p, q}^{2} f(a+t \zeta(b, a))\right|_{0} ^{r} \mathrm{~d}_{p, q} t\right)^{\frac{1}{r}} \\
& \quad \leq \frac{p q^{2} \zeta^{2}(b, a)}{p+q}\left(\int_{0}^{1} t^{s}(1-q t)_{0} \mathrm{~d}_{p, q} t\right)^{\frac{1}{s}}
\end{aligned}
$$




$$
\begin{aligned}
& \times\left(\left|{ }_{a} \mathcal{D}_{p, q}^{2} f(a)\right|^{r} \int_{0}^{1}(1-t)(1-q t){ }_{0} \mathrm{~d}_{p, q} t+\left|{ }_{a} \mathcal{D}_{p, q}^{2} f(b)\right|^{r} \int_{0}^{1} t(1-q t){ }_{0} \mathrm{~d}_{p, q} t\right)^{\frac{1}{r}} \\
= & \frac{p q^{2} \zeta^{2}(b, a)}{(p+q)} \lambda^{\frac{1}{s}}\left(\frac{\left(p^{3}-p^{2}+p q^{2}+p^{2} q\right)\left|{ }_{a} \mathcal{D}_{p, q}^{2} f(a)\right|^{r}+\left.p^{2}{ }_{a} \mathcal{D}_{p, q}^{2} f(b)\right|^{r}}{(p+q)\left(q^{2}+p q+p^{2}\right)}\right)^{\frac{1}{r}} .
\end{aligned}
$$

\section{Conclusion}

We have derived a new generalized post quantum integral identity using twice $(p, q)$ differentiable functions. Utilizing this new identity as an auxiliary result, we have obtained several new post quantum estimates of upper bounds using the class of preinvex functions. We would like to mention here that if $\zeta(b, a)=b-a$, then all the main results of this paper reduce to the results for classical convex functions, and it is pertinent to mention here that these results are also new in the literature. We hope that the ideas and techniques of this paper will inspire interested readers working in this field.

\section{Acknowledgements}

The authors would like to express their sincere thanks to the editor and the anonymous reviewers for their helpful comments and suggestions.

\section{Funding}

The work was supported by the Natural Science Foundation of China (Grant Nos. 61673169, 61374086, 11401191, 11626101, 11601485) and the Natural Science Foundation of Huzhou City (Grant No. 2018YZ07).

\section{Availability of data and materials}

Not applicable.

\section{Competing interests}

The authors declare that they have no competing interests.

\section{Authors' contributions}

All authors contributed equally to the writing of this paper. All authors read and approved the final manuscript.

\section{Author details}

'Department of Mathematics, Government College (GC) University, Faisalabad, Pakistan. ${ }^{2}$ Department of Mathematics, COMSATS University Islamabad, Islamabad, Pakistan. ${ }^{3}$ Department of Mathematics, Huzhou University, Huzhou, China.

\section{Publisher's Note}

Springer Nature remains neutral with regard to jurisdictional claims in published maps and institutional affiliations.

Received: 14 March 2020 Accepted: 28 September 2020 Published online: 07 October 2020

\section{References}

1. Acar, T., Aral, A., Mohiuddine, S.A.: On Kantorovich modification of (p, q)-Baskakov operators. J. Inequal. Appl. 2016, Article ID 98 (2016)

2. Acar, T., Aral, A., Mohiuddine, S.A.: Approximation by bivariate $(p, q)$-Bernstein-Kantorovich operators. Iran. J. Sci. Technol. Trans. Sci. 42, 655-662 (2018)

3. Alp, N., Sarıkaya, M.Z., Kunt, M., Işcan, I.: q-Hermite Hadamard inequalities and quantum estimates for midpoint type inequalities via convex and quasi-convex functions. J. King Saud Univ., Sci. 30(2), 193-203 (2018)

4. Awan, M.U., Talib, S., Kashuri, A., Noor, M.A., Chu, Y.-M.: Estimates of quantum bounds pertaining to new $q$-integral identity with applications. Adv. Differ. Equ. 2020, Article ID 424 (2020). https://doi.org/10.1186/s13662-020-02878-5

5. Ben-Israel, A., Mond, B.: What is invexity? J. Aust. Math. Soc. Ser. B 28, 1-9 (1986)

6. Bin Jebreen, H., Mursaleen, M., Ahasan, M.: On the convergence of Lupas ( $p, q)$-Bernstein operators via contraction principle. J. Inequal. Appl. 2019, Article ID 34 (2019)

7. Chakrabarti, R., Jagannathan, R.: A ( $p, q)$-oscillator realization of two-parameter quantum algebras. J. Phys. A 24(13), L711 (1991)

8. Jackson, F.H.: On a q-definite integrals. Q. J. Pure Appl. Math. 41, 193-203 (1910)

9. Kac, V., Cheung, P.: Quantum Calculus. Springer, New York (2002)

10. Kalsoom, H., Idrees, M., Baleanu, D., Chu, Y.-M.: New estimates of $a_{1} q_{2}$-Ostrowski-type inequalities within a class of n-polynomial prevexity of function. J. Funct. Spaces 2020, Article ID 3720798 (2020)

11. Kalsoom, H., Idrees, M., Kashuri, A., Awan, M.U., Chu, Y.-M.: Some new $\left(p_{1} p_{2}, q_{1} q_{2}\right)$-estimates of Ostrowski-type integral inequalities via $n$-polynomials s-type convexity. AIMS Math. 5(6), 7122-7144 (2020)

12. Khan, A., Sharma, V.: Statistical approximation by $(p, q)$-analogue of Bernstein-Stancu operators. Azerb. J. Math. 8(2), 100-121 (2018) 
13. Khan, M.A., Mohammad, N., Nwaeze, E.R., Chu, Y.-M.: Quantum Hermite-Hadamard inequality by means of a Green function. Adv. Differ. Equ. 2020, Article ID 99 (2020). https://doi.org/10.1186/s13662

14. Kunt, M., Işcan, I., Alp, N., Sarıkaya, M.Z:: $(p, q)$-Hermite-Hadamard inequalities and $(p, q)$-estimates for midpoint type inequalities via convex and quasi-convex functions. Rev. R. Acad. Cienc. Exactas Fís. Nat., Ser. A Mat. 112(4), 969-992 (2018)

15. Liu, W.-J., Zhuang, H.-F.: Some quantum estimates of Hermite-Hadamard inequalities for convex functions. J. Appl. Anal. Comput. 7(2), 501-522 (2017)

16. Luo, C.-Y., Du, T.-S., Awan, M.U., Zhang, Y.: Estimation-type results with respect to the parameterized ( $p, q)$-integral inequalities. AlMS Math. 5(1), 568-586 (2019)

17. Mursaleen, M., Naaz, A., Khan, A.: Improved approximation and error estimations by King type ( $p, q)$-Szasz-Mirakjan Kantorovich operators. Appl. Math. Comput. 348, 175-185 (2019)

18. Nasiruzzaman, M., Mukheimer, A., Mursaleen, M.: Some Opial-type integral inequalities via $(p, q)$-calculus. J. Inequal. Appl. 2019, Article ID 295 (2019)

19. Noor, M.A., Awan, M.U., Noor, K.I.: Quantum Ostrowski inequalities for $q$-differentiable convex functions. J. Math Inequal. 10(4), 1013-1018 (2016)

20. Noor, M.A., Noor, K.I, Awan, M.U.: Some quantum estimates for Hermite-Hadamard inequalities. Appl. Math. Comput. 251, 675-679 (2015)

21. Noor, M.A., Noor, K.I., Awan, M.U.: Some quantum integral inequalities via preinvex functions. Appl. Math. Comput. 269, 242-251 (2015)

22. Sudsutad, W., Ntouyas, S.K., Tariboon, J.: Quantum integral inequalities for convex functions. J. Math. Inequal. 9(3), 781-793 (2015)

23. Tariboon, J., Ntouyas, S.K.: Quantum integral inequalities on finite intervals. J. Inequal. Appl. 2014, Article ID 121 (2014)

24. Tunç, M., Göv, E.: Some integral inequalities via (p,q)-calculus on finite intervals. RGMIA Res. Rep. Collect. 19, $95(2016)$

25. Weir, T., Mond, B.: Pre-invex functions in multiple objective optimization. J. Math. Anal. Appl. 136(1), 29-38 (1988)

26. Zhang, Y., Du, T.-S., Wang, H., Shen, Y.-J.: Different types of quantum integral inequalities via $(\alpha, m)$-convexity. J. Inequal. Appl. 2018, Article ID 264 (2018)

\section{Submit your manuscript to a SpringerOpen ${ }^{\circ}$ journal and benefit from:}

- Convenient online submission

- Rigorous peer review

- Open access: articles freely available online

- High visibility within the field

- Retaining the copyright to your article

Submit your next manuscript at $\boldsymbol{~ s p r i n g e r o p e n . c o m ~}$ 